\title{
On the expected relationship between inbreeding, fitness, and extinction
}

\author{
Konstantinos THEODOROU ${ }^{\mathrm{a} *}$, Denis COUVET ${ }^{\mathrm{b}}$ \\ a Biodiversity Conservation Laboratory, Department of Environmental Studies, University of \\ the Aegean, University Hill, 81100 Mytilene, Greece \\ ${ }^{\mathrm{b}}$ CRBPO, Muséum National d'Histoire Naturelle, 55, rue Buffon, 75005 Paris, France
}

(Received 4 August 2005; accepted 17 January 2006)

\begin{abstract}
We assessed the expected relationship between the level and the cost of inbreeding, measured either in terms of fitness, inbreeding depression or probability of extinction. First, we show that the assumption of frequent, slightly deleterious mutations do agree with observations and experiments, on the contrary to the assumption of few, moderately deleterious mutations. For the same inbreeding coefficient, populations can greatly differ in fitness according to the following: (i) population size; larger populations show higher fitness (ii) the history of population size; in a population that recovers after a bottleneck, higher inbreeding can lead to higher fitness and (iii) population demography; population growth rate and carrying capacity determine the relationship between inbreeding and extinction. With regards to the relationship between inbreeding depression and inbreeding coefficient, the population size that minimizes inbreeding depression depends on the level of inbreeding: inbreeding depression can even decrease when population size increases. It is therefore clear that to infer the costs of inbreeding, one must know both the history of inbreeding (e.g. past bottlenecks) and population demography.
\end{abstract}

inbreeding / extinction / conservation / genetic load / deleterious mutation

\section{INTRODUCTION}

Small and isolated populations are associated with high rates of inbreeding and loss of genetic diversity. There is increasing evidence that inbreeding has negative effects both on fitness and probability of extinction due to the accumulation of recurrent deleterious alleles (e.g. [24, 40]). Moreover, low genetic diversity may compromise the adaptive response of populations to a changing environment $[9,15]$ and, as a consequence, their long-term persistence.

In order to predict the effects of inbreeding on population viability, Morton et al. [34] developed a model according to which the decline in fitness due to

*Corresponding author: ktheo@aegean.gr 
inbreeding can be deduced from the current level of the inbreeding coefficient. The idea behind this approach is that neutral genetic variation, measured either by means of pedigree analysis and/or neutral genetic markers, can be a reliable predictor of the expected reduction in fitness due to inbreeding [21]. In a comprehensive meta-analysis on 170 threatened taxa, Spielman et al. [42], found that threatened species show significantly lower heterozygosity at neutral loci than non-threatened closely-related species concluding that the risk to extinction is correlated to neutral genetic variation.

The model of Morton et al. [34] is still widely used to evaluate the effects of inbreeding on the extinction of endangered populations (e.g. $[21,24,36])$. Moreover, the computer program Vortex, which is widely applied in population viability analyses, also uses the relationships derived by Morton et al. [34].

However, several observations contradict the simple relationship which is generally assumed. There is increasing evidence that the effects of inbreeding would strongly depend on parameters such as (i) the history of inbreeding; populations that have a history of inbreeding would tend to show a relatively lower decrease in fitness with further inbreeding $[4,26]$, and (ii) population size; populations with larger effective size would show lower reduction in fitness traits because of more efficient purging [10, 12,31].

However, in each of these situations, the outcome is unclear if populations are compared for the same level of inbreeding. For instance, large populations experience a lower decrease in fitness and a lower inbreeding rate than small populations. As a consequence, there might be no difference when populations with identical inbreeding level are compared. Moreover, it is important to investigate whether populations with a history of past inbreeding show a higher fitness than other populations.

The goal of our study was to investigate through numerical simulations the expected relationship between the level and the cost of inbreeding and how this depends on parameters such as population size and history as well as on the model for deleterious mutations assumed. We show that in most cases, the models traditionally used $[21,24,34,36]$ cannot accurately predict the relationship between inbreeding and cost of inbreeding.

We used three different measures to quantify the cost of inbreeding: (i) mean population fitness; this measure evaluates the effects of generalized inbreeding, which is due to the finite size of the population regardless of the mating system; (ii) inbreeding depression, which measures the decline in fitness in offspring from mating between relatives compared to the fitness of offspring from random matings within the same population [25]; we studied these parameters separately since the level of inbreeding generally affects fitness and inbreeding 
depression in a different way [2]; and (iii) the probability of extinction; we considered populations of fluctuating demography in order to directly estimate how an increase in inbreeding affects population viability. Moreover, we investigated how the relationship between inbreeding and extinction depends on population growth rate.

Finally, our study bears relationship to the issue of purging of genetic load, i.e. the decline in fitness due to the accumulation of deleterious mutations expressed as 1- mean population fitness. Although much attention has been paid to the possibility of purging the genetic load, the way in which a researcher chooses to estimate purging will affect the interpretation of the results obtained, as clearly explained in Crnokrak and Barrett [7]. The present work explores two estimates of purging, namely purging of genetic load, and purging of inbreeding depression.

\section{METHODS}

\subsection{The general model}

We considered randomly mating monoecious populations growing in discrete generations. Let $N_{t}$ denote the number of reproducing adults in generation $t$ and $w_{t}$ denote the mean population viability; viability is a function of the frequency of deleterious mutations. Populations were founded from an infinite population at mutation-selection balance (details in Appendix). The expected population size in each generation is given by the deterministic growth rate $\lambda=R \times w_{t}$, where $R$ denotes the number of progeny per reproductive individual per generation, and it is assumed to be constant. In order to assess the probability of extinction due to both genetic and demographic stochasticity, we followed Theodorou and Couvet [44]: population size is drawn from a Poisson distribution with expected value $N_{t+1}=\lambda N_{t}$ [16]. In this case, 1000 simulations were run for each parameter set and the probability of survival was given by the average of the replicate simulations. If the resulting size is above carrying capacity, $K$, population size is truncated to $K$. This form of densitydependence is a time-discrete version of the ceiling model. Such crude representation of population regulation yields first insights into the effect of stochastic factors on the extinction risk. Different density-dependent models which lead to different relationships between population extinction and growth rate (see review in [17]) might be used in the future to explore the generality of our results. 


\subsection{Genetic model}

We considered a two alleles model, $A$ being the wild-type allele and $a$ an unconditionally deleterious and partially recessive allele. The three diploid genotypes $A A, A a$, aa have a relative fitness of $1,1-h s, 1-s$ respectively; $h$ measures the degree of dominance of the deleterious mutant and $s$ the magnitude of the deleterious effect. Mutation towards the mutant allele, $a$, happens at a constant rate, $u$, per gamete and per generation while back mutations are ignored. Details for the calculation of mean allele frequency, fitness and inbreeding depression are given in the appendix.

Our model assumes that fitness across loci is multiplicative and that overall mean fitness due to slightly deleterious and lethal alleles is the product of fitness values for each class of mutations separately [47].

We also examined genetic variability for neutral loci. Assuming that neutral loci are not linked to loci where deleterious alleles are present, the inbreeding coefficient $f_{t}$ at each generation $t$ is given by the equation

$$
f_{t+1}=(1-u)^{2}\left\{\frac{1}{2 N_{t}}+\left(1-\frac{1}{2 N_{t}}\right) f_{t}\right\} \text {. }
$$

\subsection{Mutation parameters}

Values for mutation parameters are still unknown for most organisms. Most available results come from experiments conducted on the Drosophila species. Several experiments suggest that new mutations are in majority slightly deleterious (mean selection coefficient $\bar{s}=0.01-0.03$ ), with mutation rates per diploid genome of $U=1$ and a mean dominance coefficient of $\bar{h}=0.2-0.4$ (see review in [29]).

However, the validity of these estimates has recently been questioned; new experimental studies on Drosophila melanogaster and other organisms suggest that mutation rates are much lower, $U=0.01-0.02$, and with a higher effect on fitness than previously predicted, $\bar{s}=0.1-0.2[1,23]$.

In this study we investigated two sets of parameters for deleterious mutations (Tab. I). The selection coefficient, $s$, is assumed to follow a gamma distribution with mean $\bar{s}$,

$$
g(s)=(\beta / \bar{s})^{\beta} s^{\beta-1} e^{-s \beta / \bar{s}} / \Gamma(\beta)
$$

with shape parameters $\beta=1$ (exponential distribution) and $\beta=2$ for each model respectively, in accordance with the analysis of Keightley [22], 
Table I. Sets of parameters for slightly deleterious mutations*.

\begin{tabular}{ccccc}
\hline & $\mathrm{U}$ & $\bar{s}$ & $\bar{h}$ & $\beta$ \\
\hline Model I & 1 & 0.02 & 0.35 & 1 \\
Model II & 0.1 & 0.2 & 0.2 & 2 \\
\hline
\end{tabular}

* For lethals, $s$ follows a uniform distribution in the range $[0.9,1] ; h=0.02, U=0.03$. $\mathrm{U}$ : mutation rate per diploid genome.

$\bar{s}$ : mean selection coefficient.

$\bar{h}$ : mean dominance coefficient.

$\beta$ : shape parameters.

García-Dorado and Caballero [18] and García-Dorado et al. [19]. The dominance coefficient is related to the selection coefficient with the relationship $h=\exp (-k s) / 2$, where $k$ is chosen so as the average dominance coefficient to be the desired $\bar{h}$.

To examine the effects of variables $h$ and $s$ by means of the transition matrix approach, we followed the approach of Couvet [6]. For lethal mutations, the selection coefficient was assumed to follow a uniform distribution in the range $[0.9,1] ; h=0.02, U=0.03$.

Fitness values for moderately deleterious and lethal mutations were obtained separately and their product gave the overall fitness [47].

\section{RESULTS}

In our results, mean population fitness, inbreeding depression and survival probability are shown in relation to the inbreeding coefficient and not time, as is often the case. This allowed us to directly investigate the relationship between fitness and neutral variation and the extent of purging with inbreeding, which are important goals for the conservation of endangered populations.

\subsection{Influence of population size}

We first investigated the relationship between the inbreeding coefficient, $f$, and the mean population fitness in stable populations of $N=10,50$ or 100 individuals.

If the recurrent deleterious mutations follow model I (high mutation rate slightly deleterious alleles), fitness decreases monotonically with the level of inbreeding (Fig. 1a). However, for a given inbreeding coefficient, fitness varied dramatically with population size, with larger populations always showing 
(a)

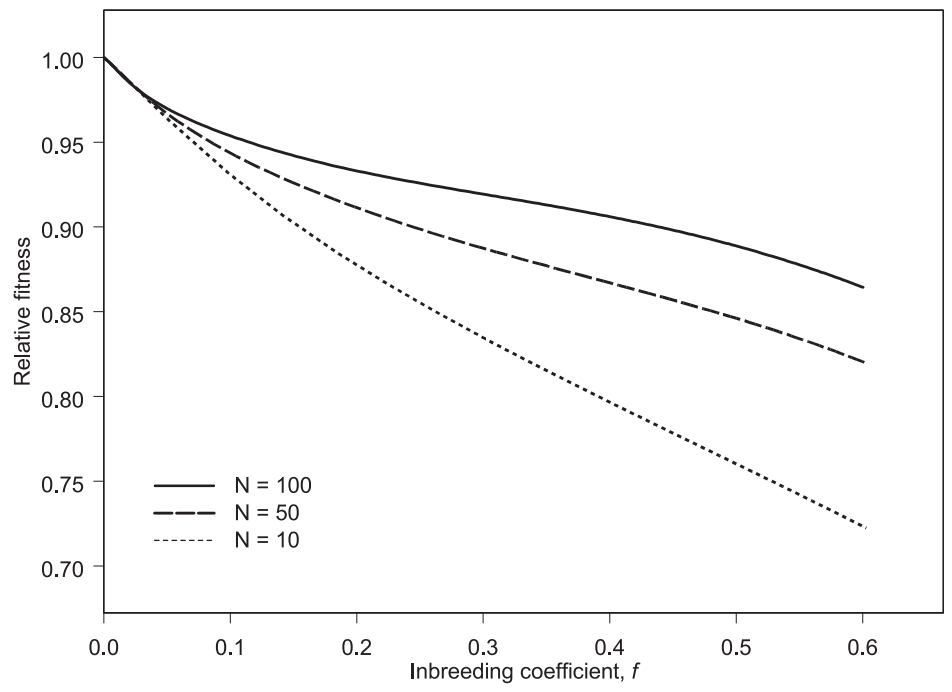

(b)

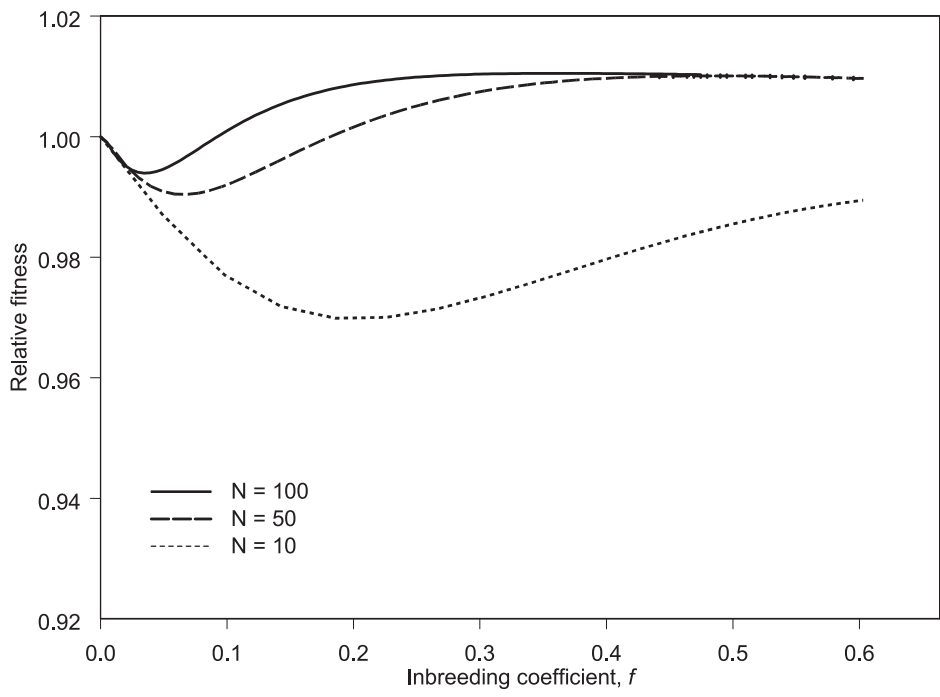

Figure 1. Relationship between fitness and inbreeding coefficient for different population sizes. The mean fitness of the population was scaled by the mean fitness of an infinitely large population with identical $f$ in order to visualize the decrease in fitness due to mutation accumulation. Values of mutation parameters were chosen according to (a) model I (mildly deleterious - high mutation rate) and (b) model II (moderately deleterious - low mutation rate). 


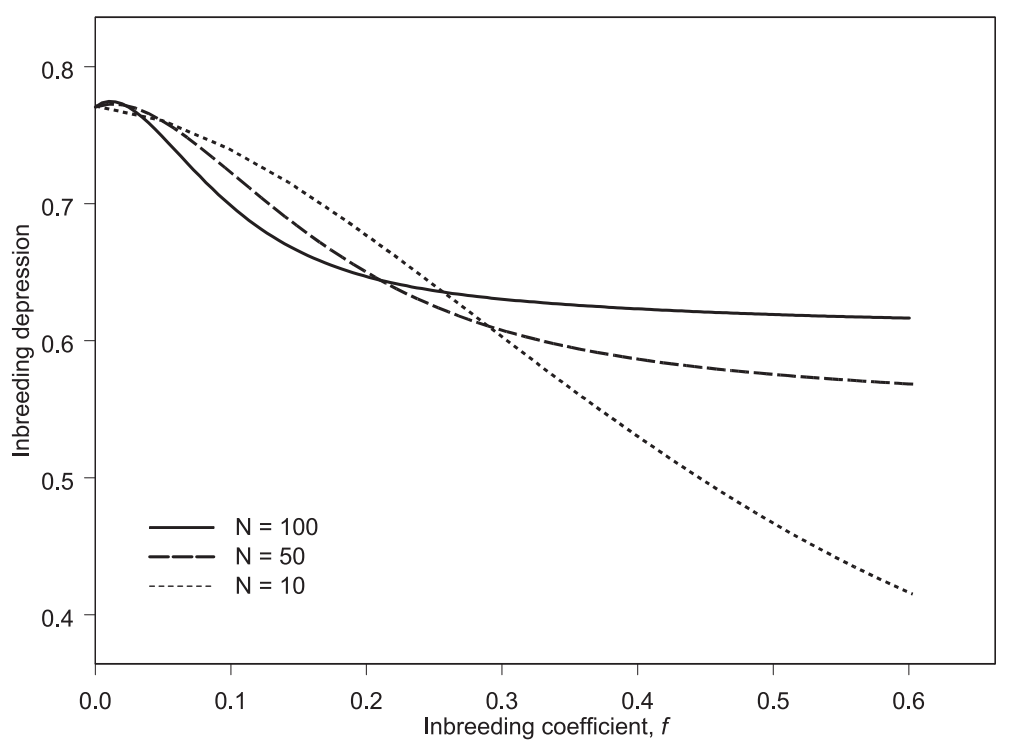

Figure 2. Relationship between inbreeding depression and inbreeding coefficient for different population sizes. Values of mutation parameters were chosen according to model I.

a higher fitness. In large populations, genetic drift was weaker and selection against deleterious mutations was more effective than in small populations. Hence, large populations have a greater potential to purge deleterious alleles.

According to model II (low mutation rates - moderately deleterious alleles), higher inbreeding coefficients may be associated to higher fitness (Fig. 1b). The high homozygosity due to inbreeding exposes partially recessive deleterious alleles and thus makes selection more efficient. During the first generations, we observed a small decline in fitness associated to the process of purging. For higher inbreeding coefficients, populations had already purged the majority of the load and fitness rebounds to levels even higher than in infinite populations. Notice that model II yields results that were in disagreement with the majority of observations in natural populations [7,38]. Hereafter, we show results only for simulations run under model I.

For low inbreeding coefficients, inbreeding depression decreased when population size increased (Fig. 2). However, for higher inbreeding coefficients, this relationship was reversed. In the long term, larger populations retained both higher fitness and higher levels of inbreeding depression. Notice that the relationship between inbreeding depression and the inbreeding coefficient did not depend on the set of mutation parameters used (results not shown). 


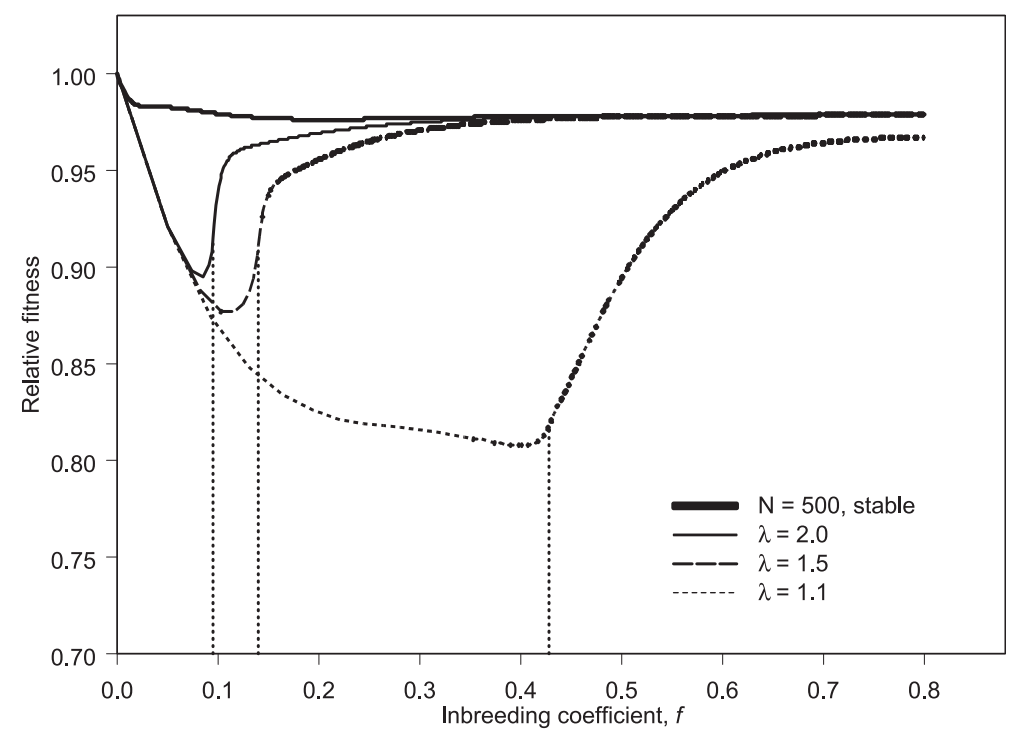

Figure 3. Relationship between fitness and inbreeding coefficient for populations that recover after a bottleneck. The fitness of a population of stable size $(N=500)$ was compared with the fitness of populations with different growth rates, $\lambda$, that recover after an initial bottleneck of $N_{0}=10$ until they reach carrying capacity, $K=500$. The vertical dotted lines indicate when populations reach carrying capacity. The values of mutation parameters were chosen according to model I.

\subsection{Influence of population history on fitness}

We compared fitness values for a population of stable size (equal to carrying capacity, $N=K=500$ ) and for populations that attained the same carrying capacity after passing through a bottleneck (initial size, $N_{0}=10$ ). We observed opposite relationships between fitness and inbreeding. An increase in the inbreeding coefficient $f$, resulted in (i) a slight decrease in the fitness of the stable population, and (ii) an increase in mean fitness when populations that recover after a bottleneck reach carrying capacity (see dashed vertical lines in Fig. 3).

After a bottleneck, the population growth rate significantly determined the relationship between fitness and inbreeding coefficient (Fig. 3). In slowergrowing populations, fitness decreased for a larger number of generations (and range of inbreeding coefficients) and only partially recovered in later generations. 


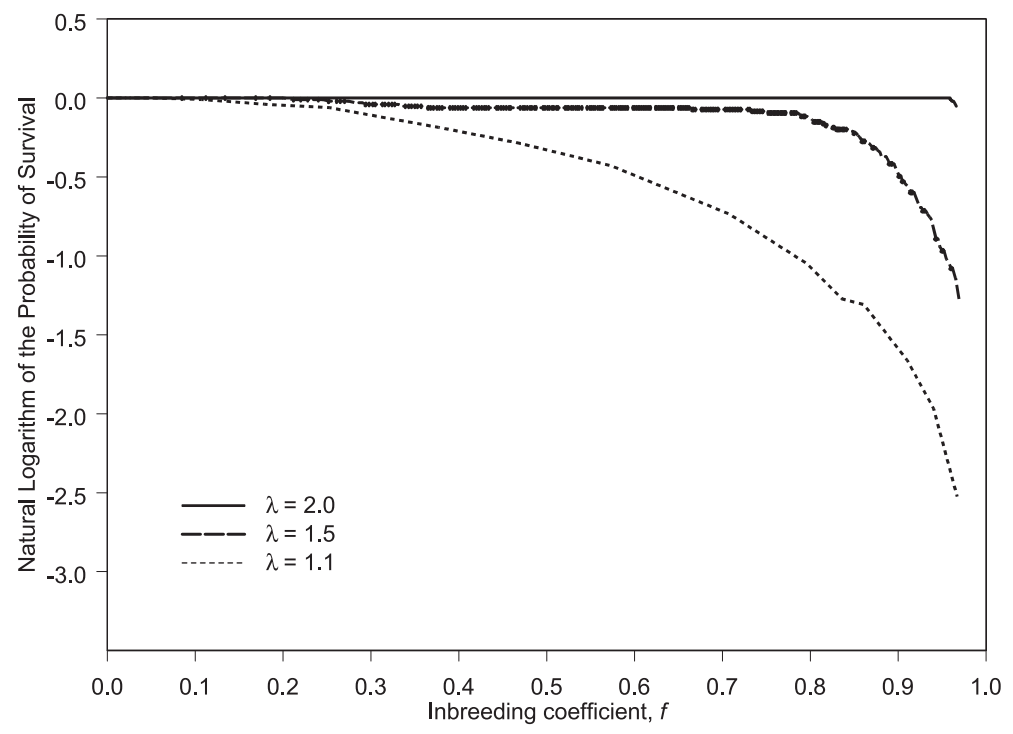

Figure 4. Relationship between probability of survival and inbreeding coefficient for populations of different growth rates, $\lambda$. The initial population size was set to $N_{0}=10$ and the carrying capacity was set to $K=100$. Values of mutation parameters were chosen according to model I.

\subsection{Relationship between extinction probability and inbreeding coefficient}

We investigated how the probability of extinction - due to both genetic and demographic stochasticity - is influenced by the level of inbreeding. In order to compare our results with previous ones (e.g. [14,40]), the natural logarithm of the probability of survival was plotted against the inbreeding coefficient.

The relationship between survival and inbreeding depends both on the carrying capacity and the population growth rate. In populations with low growth rates $(\lambda=1.1$ to 1.5$)$, there exists a threshold for the inbreeding coefficient above which the probability of extinction dramatically increases; this threshold significantly depends on the population growth rate. However, for high population growth rates $(\lambda=2)$, extinction is virtually independent of the inbreeding coefficient (Figs. 4 and 5).

When model II was considered (moderately deleterious - low mutation rate) the probability of survival was independent of the level of inbreeding irrespective of population demographic parameters (results not shown). 


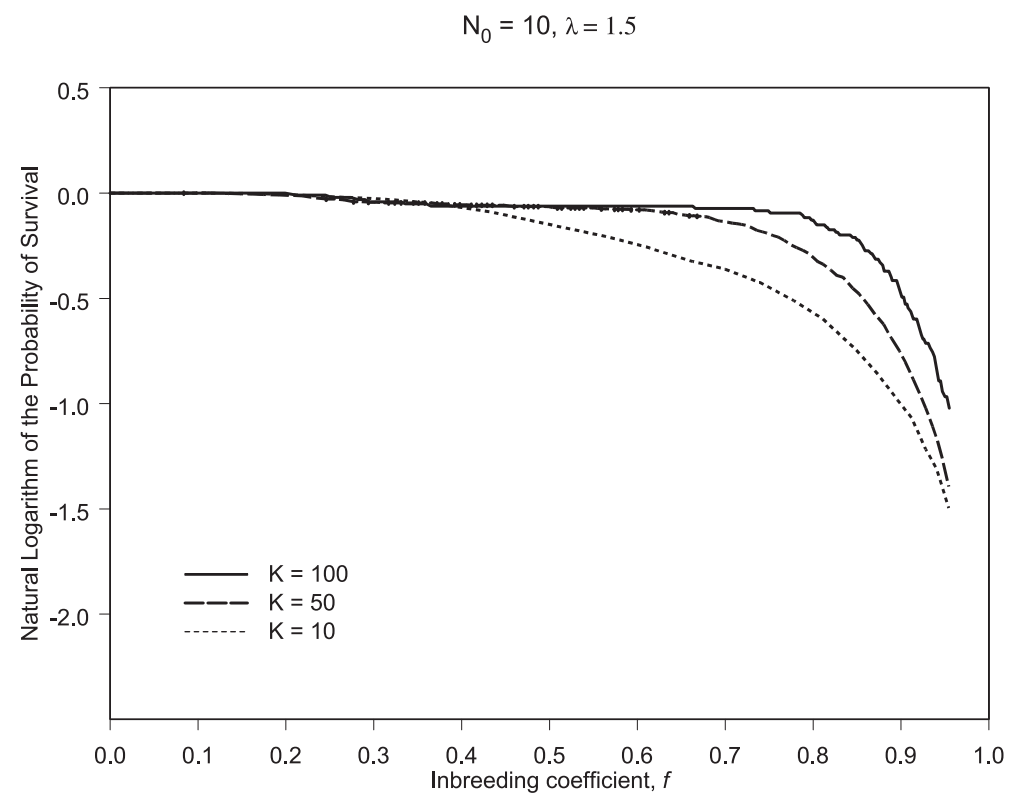

Figure 5. Relationship between probability of survival and inbreeding coefficient for different carrying capacities, $\mathrm{K}$. The initial population size was set to $N_{0}=10$ and the growth rate was set to $\lambda=1.5$. Values of mutation parameters were chosen according to model I.

\section{DISCUSSION}

Understanding the relationship between population viability and inbreeding level is central in conservation genetics. For instance, there is still some controversy as to whether higher inbreeding, which is usually associated with small populations, results in a significant decrease in population fitness or on the contrary leads to more efficient purging of genetic load. In the latter case, deleterious mutations would not always represent a serious threat for the persistence of inbred populations. There is now increasing evidence that the former outcome is the most often found for a variety of organisms (see review in $[7,24])$.

The relationship between the level of inbreeding and population fitness has motivated substantial experimental work and several models have been proposed for that purpose (see review in $[21,24]$ ). It is interesting to note for instance that the program "Vortex", which is widely used for population viability analyses, assumes that the decline in fitness can be deduced by the current level of inbreeding, i.e. $w_{I}=w_{O} e^{-B f}$, with $w_{I}, w_{O}$ being the fitness of inbred and outcrossed progeny respectively [33]. 
However, our results suggest that in most cases this relationship would fail to predict the extent of genetic load in a population.

\subsection{Relationship between fitness and inbreeding}

We showed that population fitness depends on the rate of increase of inbreeding, which is governed by the effective population size. Hence, for the same level of inbreeding, larger populations show higher fitness. The dependence of fitness on the rate of increase of inbreeding rather than on the actual level of inbreeding is a result found in several experimental studies (e.g. $[10,31])$.

More interestingly, a positive relationship between fitness and inbreeding level can occur. In contrast to populations of stable size, populations that rebound after a bottleneck show higher fitness as the inbreeding coefficient increases. Notice that such an increase has indeed been observed experimentally $[13,32]$.

As a consequence, two populations of the same size and level of inbreeding may show different levels and trends in fitness. Thus, if one ignores the history of the population, this observation could lead to erroneous conclusions, such as stating that inbreeding leads to efficient purging, which is true only under restricted conditions [20].

Our study explored purging in finite panmictic populations. This kind of purging process must be distinguished from inbreeding due to systematic nonrandom mating. Glémin [20] showed that deleterious mutations can be purged through nonrandom mating regardless of their dominance coefficient while only highly recessive mutations can be purged in the panmictic case. Thus, experimental studies that perform intentional inbreeding (e.g. full-sib mating) in order to study purging by inbreeding will overestimate the efficiency of selection in the case of small panmictic populations.

Our analysis offers an alternative explanation to the substantial variation detected in empirical studies reporting on the relationship between inbreeding levels and demographic performances of populations, ranging from very negative to positive in some cases. Although this variation is commonly attributed to the stochastic nature of inbreeding or to small experimental sample sizes $[15,21]$, our results suggest that it could also be due to genuine differences associated with past population demography.

Hence, genetic methods for inferring recent changes in effective population size $[3,45,46]$ could be very useful in order to provide a reasonable estimate of the extent of genetic load and assess, therefore, the potential positive impact on 
fitness of introducing unrelated individuals in the populations. Such beneficial effects have been observed, for instance, for the adder [30] and the great-prairie hen [48]. However, given the cost of such measures, as well as the negative effects associated with the loss of characters specific to local populations, it is important to predict the extent of these benefits.

Our results are sensitive to the values considered for mutation parameters. If genetic load is due to moderately deleterious alleles (referred to as model II in our study), inbreeding would remove the greatest part of these alleles and populations would retain high levels of fitness. However, purging does not seem to be the rule in natural populations $[4,40]$. There is an overall positive relationship between genetic load and inbreeding coefficient [14, 39]. Thus, based on these observations, our results suggest that model I might be closer to reality than model II.

\subsection{Relationship between inbreeding depression and population size}

In populations that undergo several generations of inbreeding due to finite size, genetic load increases due to the accumulation of deleterious alleles. In contrast, inbreeding depression decreases since the fitness between outcrossed and inbred progeny becomes similar as inbreeding goes on (Fig. 2). At equilibrium, inbreeding depression is expected to increase with population size, because higher genetic variance is conserved within these populations [2].

However, when populations are at demographic disequilibrium, the population size that minimizes inbreeding depression depends on the level of inbreeding. For low inbreeding coefficients - following, for instance, a bottleneck smaller populations show higher inbreeding depression than larger ones. In contrast, in subsequent generations, and therefore higher inbreeding coefficients, inbreeding depression is greater in large populations. Consequently, one must know both the present inbreeding coefficient and previous changes in population size to predict the extent of inbreeding depression.

\subsection{Relationship between population survival and inbreeding}

A crucial issue in conservation genetics is whether an increase in the level of inbreeding, is associated with a higher extinction probability. Frankham [14] suggested that a threshold relationship exists between survival and inbreeding level. However, our findings suggest that the threshold value varies with population demography. In other words, a same threshold will be observed only for populations of similar demography (as in [14]). 
Moreover, for high growth rates (e.g. $\lambda=2$ ), and large population size, such a threshold disappears, and a high survival probability is ensured regardless of the level of inbreeding (independent relationship sensu Frankham [14]). Population persistence is strongly influenced by the population growth rate and negative trends in growth rate due to inbreeding have been shown for several natural populations $[35,37,41]$.

Overall, our study suggests that the relationship between the level and the cost of inbreeding, expressed either as a decrease in mean fitness, inbreeding depression or probability of extinction is highly dependent on the demographic parameters of the populations under study, i.e. population size and growth rate, and the history of the population (e.g. past bottlenecks).

\section{REFERENCES}

[1] Bataillon T.M., Estimation of spontaneous genome-wide mutation rate parameters: whither beneficial mutations?, Heredity 84 (2000) 497-501.

[2] Bataillon T., Kirkpatrick M., Inbreeding depression due to mildly deleterious mutations in finite populations: size does matter, Genet. Res. 75 (2000) 75-81.

[3] Beaumont M.A., Estimation of population growth or decline in genetically monitored populations, Genetics 164 (2003) 1139-1160.

[4] Byers D.L., Waller D.M., Do plant populations purge their genetic load? Effects of population size and mating history on inbreeding depression, Ann. Rev. Ecol. System. 30 (1999) 479-513.

[5] Charlesworth D., Charlesworth B., Inbreeding depression and its evolutionary consequences, Annu. Rev. Ecol. Syst. 18 (1987) 237-268.

[6] Couvet D., Deleterious effects of restricted gene flow in fragmented populations, Conserv. Biol. 16 (2002) 369-376.

[7] Crnokrak P., Barrett S.C.H., Perspective: Purging the genetic load: a review of the experimental evidence, Evolution 56 (2002) 2347-2358.

[8] Crow J.F., Kimura M., An Introduction to Population Genetics Theory, 1st edn., Alpha Editions, Minneapolis, 1970.

[9] Day S.B., Bryant E.H., Meffert L.M., The influence of variable rates of inbreeding on fitness, environmental responsiveness, and evolutionary potential, Evolution 57 (2003) 1314-1324.

[10] Ehiobu N.G., Goddard M.E., Taylor J.F., Effect of rate of inbreeding on inbreeding depression in Drosophila melanogaster, Theor. Appl. Genet. 77 (1989) 123-127.

[11] Ewens W.J., Mathematical population genetics, 1st edn., Springer, New York, 1979.

[12] Fischer M., Matthies D., RAPD variation in relation to population size and plant fitness in the rare Gentianella germanica (Gentianaceae), Am. J. Bot. 85 (1988) 811-819. 
[13] Fowler K., Whitlock M.C., The variance in inbreeding depression and the recovery of fitness in bottlenecked populations, P. Roy. Soc. Lond. B Bio 266 (1999) 2061-2066.

[14] Frankham R., Inbreeding and extinction: a threshold effect, Conserv. Biol. 9 (1995) 792-799.

[15] Frankham R.J., Ballou D., Briscoe DA, Introduction to Conservation Genetics, Cambridge University Press, Cambridge, 2002.

[16] Gabriel W., Bürger R., Survival of small populations under demographic stochasticity, Theor. Popul. Biol. 41 (1992) 44-71.

[17] Gabriel W., Ferrière R., From individual interactions to population viability, in: Ferrière R., Dieckmann U., Couvet D. (Eds.), Evolutionary Conservation Biology, Cambridge University Press, Cambridge, 2003, pp. 19-40.

[18] García-Dorado A., Caballero A., On the average coefficient of dominance of deleterious spontaneous mutations, Genetics 155 (2000) 1991-2001.

[19] García-Dorado A., López-Fanjul C., Caballero A., Properties of spontaneous mutations affecting quantitative traits, Genet. Res. 74 (1999) 341-350.

[20] Glémin S., How are deleterious mutations purged? Drift versus nonrandom mating, Evolution 57 (2003) 2678-2687.

[21] Hedrick P.W., Kalinowski S.T., Inbreeding depression in conservation biology, Annu. Rev. Ecol. System. 31 (2000) 139-162.

[22] Keightley P.D., The distribution of mutation effects on viability in Drosophila melanogaster, Genetics 138 (1994) 1315-1322.

[23] Keightley P.D., Eyre-Walker A., Terumi Mukai and the riddle of deleterious mutation rates, Genetics 153 (1999) 515-523.

[24] Keller L.F., Waller D.M., Inbreeding effects in wild populations, Trends Ecol. Evol. 17 (2002) 230-241.

[25] Kirkpatrick M., Jarne P., The effects of a bottleneck on inbreeding depression and the genetic load, Am. Nat. 155 (2000) 154-167.

[26] Lacy R.C., Ballou J.D., Effectiveness of selection in reducing the genetic load in populations of Peromyscus polionotus, Evolution 50 (1998) 2187-2200.

[27] Lande R., Schemske W., The evolution of self-fertilization and inbreeding depression in plants. I. Genetic models, Evolution 39 (1985) 24-40.

[28] Lynch M., Conery J., Bürger R., Mutational meltdowns in sexual populations, Evolution 49 (1995) 1067-1080.

[29] Lynch M., Blanchard J., Houle D., Kibota T., Schultz S., Vassilieva L., Willis J., Perspective: spontaneous deleterious mutation, Evolution 53 (1999) 645-663.

[30] Madsen T., Shine R., Olsson M., Wittzell H., Restoration of an inbred adder population, Nature 402 (1999) 34-35.

[31] Matthies D., Bräuer I., Maibom W., Tcsharntke T., Population size and the risk of local extinction: empirical evidence from rare plants, Oikos 105 (2004) 481-488.

[32] Miller P.S., Hedrick P.W., Purging of inbreeding depression and fitness decline in bottlenecked populations of Drosophila melanogaster, J. Evol. Biol. 14 (2001) 595-601.

[33] Miller P.S., Lacy R.C., VORTEX: a stochastic simulation of the extinction process. Version 9.21 user's manual, Conservation Breeding Specialist Group (SSC/IUCN), Apple Valley, MN, 2003. 
[34] Morton N.E., Crow J.F., Muller H.J., An estimate of the mutational damage in man from data on consanguineous marriages, Proc. Natl. Acad. Sci. USA 42 (1956) 855-863.

[35] O'Grady J.J., Reed D.H., Brook B.W., Frankham R., What are the best correlates of predicted extinction risks? Biol. Conserv. 118 (2004) 513-520.

[36] Ralls K., Ballou J.D., Templeton A., Estimates of lethal equivalents and the cost of inbreeding in mammals, Conserv. Biol. 2 (1988) 185-193.

[37] Reed D.H., Extinction risk in fragmented habitats, Anim. Conserv. 7 (2004) 181-191.

[38] Reed D.H., Relationship between population size and fitness, Conserv. Biol. 19 (2005) 563-568.

[39] Reed D.H., Frankham R., Correlation between fitness and genetic diversity, Conserv. Biol. 17 (2003) 230-237.

[40] Reed D.H., Lowe E.H., Briscoe D.A., Frankham R., Inbreeding and extinction: effects of rate of inbreeding, Conserv. Genet. 4 (2003) 405-410.

[41] Reed D.H., O'Grady J.J., Brook B.W., Ballou J.D., Frankham R., Estimates of minimum viable sizes for vertebrates and factors influencing those estimates, Biol. Conserv. 113 (2003) 23-34.

[42] Spielman D., Brook W.D., Frankham R., Most species are not driven to extinction before genetic factors impact them, Proc. Natl. Acad. Sci. USA 101 (2004) 15261-15264.

[43] Theodorou K., Couvet D., Inbreeding depression and heterosis in a subdivided population; influence of the mating system, Genet. Res. 80 (2002) 107-116.

[44] Theodorou K., Couvet D., Introduction of captive breeders to the wild: harmful or beneficial?, Conserv. Genet. 5 (2004) 1-12.

[45] Vitalis R., Couvet D., Estimation of effective population size and migration rate from one- and two-locus identity measures, Genetics 157 (2001) 911-925.

[46] Wang J., Whitlock M.C., Estimating effective population size and migration rates from genetic samples over space and time, Genetics 163 (2003) 429-446.

[47] Wang J., Caballero A., Keightley P.D., Hill W.G., Bottleneck effect on genetic variance: a theoretical investigation of the role of dominance, Genetics 150 (1998) 435-447.

[48] Westemeier R.L., Brawn J.D., Simpson S.A., Esker T.L., Jansen R.W., Walk J.W., Kershner E.L., Bouzat J.L., Paige K.N., Tracking the long-term decline and recovery of an isolated population, Science 282 (1998) 1695-1698.

\section{APPENDIX. CALCULATION OF ALLELE FREQUENCY, FITNESS AND INBREEDING DEPRESSION}

\section{Computing allele frequency}

In order to calculate allele frequency and, thereby, population fitness in each generation, we used a transition-matrix approach $[11,28,43]$. According to this model:

$$
\mathbf{Q}_{t+1}=\mathbf{P} \mathbf{Q}_{t}
$$


where $\mathbf{Q}_{t}=\left[q_{0, t} \ldots q_{i, t} \ldots q_{2 N t, t}\right]^{T}$ is the $\left(2 N_{t}+1\right) \times 1$ column matrix that describes the probability distribution of the states of the mutant allele $a$ at a given locus in generation $t$. For any locus, there are $2 N_{t}+1$ possible states ranging for complete loss $(i=0)$ to complete fixation $\left(i=2 N_{t}\right) . \mathbf{P}=\left[p_{i j}\right]$ is the $\left(2 N_{t+1}+1\right) \times\left(2 N_{t}+1\right)$ matrix of transition probabilities. Each element, $p_{i j}$, of this matrix is the probability of having $i$ mutant alleles in generation $t+1$, given $j$ mutant alleles in generation $t$.

\section{Computing the elements of the transition-matrix, $p_{i j}$}

We assume that allelic frequency in generation $t$ is $q_{j t}=j / 2 \mathrm{~N}_{t}, j=0$, $\ldots, 2 \mathrm{~N}_{t}$, where $N_{t}$ is the population size in generation $t$. After mutation the frequency of the mutant allele $a$ becomes

$$
q_{j}^{\prime}=u\left(1-q_{j t}\right) .
$$

The corresponding genotypic frequencies under random mating system are

$$
\begin{gathered}
P_{j}^{\prime}=\left(1-q_{j}^{\prime}\right)^{2} \\
H_{j}^{\prime}=2\left(1-q_{j}^{\prime}\right) q_{j}^{\prime} \\
R_{j}^{\prime}=q_{j}^{\prime 2}
\end{gathered}
$$

After selection, genotypic frequencies are modified as follows:

$$
\begin{gathered}
P_{j}=P_{j}^{\prime} / \bar{w} \\
H_{j}=H_{j}^{\prime}(1-h s) / \bar{w} \\
R_{j}=R_{j}^{\prime}(1-s) / \bar{w}
\end{gathered}
$$

where $\bar{w}$ is the mean fitness of the population.

The frequency of the mutant allele is therefore

$$
q_{j}=R_{j}+H_{j} / 2 .
$$

The elements of the transition matrix, $p_{i j}$, can be calculated from the binomial probability expression. The transition probability from a state with $j$ mutant alleles (among the $N_{t}$ individuals) at a locus in generation $t$ to a state with $i$ mutant alleles in generation $t+1$ (among $N_{t+1}$ individuals) is

$$
p_{i j}=\left(\begin{array}{l}
2 N_{t+1} \\
i
\end{array}\right) q_{j}^{i}\left(1-q_{j}\right)^{2 N_{t+1}-i} .
$$




\section{Fitness and inbreeding depression}

The mean fitness of the population at a selected locus is computed as in [43]:

$$
\bar{w}_{x}=1-h s H-s R,
$$

where

$$
\begin{gathered}
H=\sum_{i=0}^{2 N} 2 \frac{i}{2 N}\left(1-\frac{i}{2 N}\right) q_{i} \\
R=\sum_{i=0}^{2 N}\left(\frac{i}{2 N}\right)^{2} q_{i} .
\end{gathered}
$$

Fitness is assumed to be multiplicative across loci; the average fitness over the whole genome is simply the product of the fitness at each locus [8]:

$$
\bar{W}_{x}=\prod_{i=1}^{L} \bar{w}_{x},
$$

where $L$ is the number of loci. When more than one class of mutants is considered, e.g. detrimentals of small effects on fitness and lethals, total fitness is $\bar{W}=\prod \bar{W}_{i}$, with $W_{i}$ being the fitness over all loci for the mutant class $i$.

Inbreeding depression is defined as the proportional decrement in fitness of inbred individuals, $\bar{w}_{x}$, compared to the fitness of outbred individuals, $\bar{w}_{x}$, $\delta=1-\bar{w}_{s} / \bar{w}_{x}[5,27]$. We can define $\bar{w}_{s}$ as the fitness of progeny produced by selfing:

$$
\bar{w}_{s}=1-h s H_{s}-s R_{s},
$$

where $H_{s}=\frac{H}{2}$ and $R_{S}=R+\frac{H}{4}$. With $H, R$ derived from equations (A.8a, b).

Notice that this approach may be extended to populations where no selfing occurs by considering full-sib mating instead.

\section{Initial conditions}

We considered populations issued from an infinite population under mutation-selection balance. The equilibrium frequency $(q)$ of the mutant allele in the ancestral population was obtained as the unique solution (in $[0,1]$ ) of the equation $s(1-2 h) q^{2}+s h(1+u) q-u=0$ [8]. Hence, the probability distribution of the states of the mutant allele $a$ at a given locus in generation 0 is $q_{i 0}=\left(\begin{array}{l}2 N_{o} \\ i\end{array}\right) q^{i}(1-q)^{2 N_{0}-i}$, where $i=0, \ldots, 2 N_{0}$. 